\title{
Incidental durotomy in lumbar spine surgery - a three-nation survey to evaluate its management
}

\author{
Oliver P. Gautschi • Martin N. Stienen • Nicolas R. Smoll • \\ Marco V. Corniola $•$ Enrico Tessitore $•$ Karl Schaller
}

Received: 22 May 2014 / Accepted: 8 July 2014 / Published online: 22 July 2014

(C) Springer-Verlag Wien 2014

\begin{abstract}
Background Although it is generally accepted that incidental durotomies (ID) should be primarily repaired, the current literature shows no consensus regarding the peri- and postoperative management in case of ID during lumbar spine surgery. Because ID is a rather frequent complication and may be associated with significant disability, we were interested to analyze the current handling of ID in three European countries.

Methods In March 2014, members of the Swiss, German, and Austrian neurosurgical and spine societies were asked to complete an online questionnaire regarding the management of ID during and after lumbar spine surgery. Two, respectively 4 weeks after the first invitation, reminder requests were sent to all invitees, who had not already responded at that time. Results There were 175 responses from 397 requests $(44.1 \%)$. Responders were predominantly neurosurgeons (89.7\%; $10.3 \%$ were orthopedic surgeons), of which 45.7 , 40.0 , and $17.8 \%$ work in a non-university hospital, university hospital, and private clinic, respectively. As for the perioperative management of ID, $19.4 \%$ of the responders suggest only bed rest, while, depending on the extent of the ID, $84.0 \%$ suggest additional actions, TachoSil/Spongostan with fibrin glue or a similar product and single suture repair being the
\end{abstract}

O. P. Gautschi $(\varangle) \cdot$ M. V. Corniola $\cdot$ E. Tessitore $\cdot$ K. Schaller Département de Neurosciences cliniques, Service de Neurochirurgie, Faculté de Médecine, Hôpitaux Universitaires de Genève, Rue Gabrielle-Perret-Gentil 4, 1211 Genève 14, Switzerland e-mail: ogautschi@gmail.com

M. N. Stienen

Department of Neurosurgery, Kantonsspital St. Gallen, St. Gallen, Switzerland

N. R. Smoll

Department of Medicine, Frankston Hospital, Melbourne, VIC, Australia most mentioned. Concerning epidural wound drainage in case of ID, 37.2 \% desist from placing an epidural wound drainage with or without aspiration, $30.9 \%$ place it sometimes, and $33.7 \%$ place it regularly, but only without aspiration. Most responders prescribe bed rest for $24(34.9 \%)$ or $48 \mathrm{~h}(28.0 \%)$, with much fewer prescribing bed rest for $72 \mathrm{~h}(6.3 \%)$ and none more than $72 \mathrm{~h}$, and $14.9 \%$ of participants never prescribe bed rest. The vast majority of physicians $(82.9 \%, n=$ 145 ) always inform their patients after the operation in case of ID.

Conclusions There is substantial heterogeneity in the management of incidental durotomies. The majority of spine surgeons today aim at complete/sufficient primary repair of the ID with varying recommendations concerning postoperative bed rest. Still, there is a trend towards early mobilization if the incidental durotomy has been closed completely/sufficiently with no participant favoring bed rest for more than $72 \mathrm{~h}$.

Keywords Incidental durotomy · Management · Bed rest . Treatment algorithm $\cdot$ Three-nation survey

\section{Introduction}

Incidental durotomy (ID) is a common complication of lumbar spine surgery with an incidence ranging from less than $1 \%$ to $17 \%$ varying according to the series reviewed as well as the type of surgical procedure [1, 2]. Tafzal and Sell reported in their prospective study with 1,549 patients across 14 institutions in the United Kingdom an incidence rate of $3.5 \%$ for primary discectomy, $8.5 \%$ for spinal stenosis surgery, and $13.2 \%$ for revision discectomy [3]. Other authors confirmed an increased incidence in case of revision surgery [4-9]. Furthermore, higher incidences were associated with increased patient age, presence of juxtafacet cysts, and less experience of the surgeon [7, 10-12]. In case of first-time 
operations and in experienced hands, ID is less frequently encountered. Non-treated IDs can lead to serious constraint of the patient as it may be associated with a number of symptoms and complications such as postural headache (spontaneous intracranial hypotension due to spinal cerebrospinal fluid (CSF) leakage), nausea and vomiting, photophobia, tinnitus and dizziness, persistent CSF fistula, pseudomeningocele formation, nerve root herniation with or without subsequent radicular pain and neurological deficit, subdural hematoma, delayed wound healing, and wound infection up to arachnoiditis or meningitis [13]. Although an ID is, if recognized and treated accordingly, usually self-limiting and the long-term outcome usually not affected, a persistent CSF leakage may have a multitude of unwanted sequelae for the patients $[8,10,13-17]$. Therefore, careful dissection using meticulous microscopic techniques is of paramount importance to prevent from ID and its possible negative impact on the patient recovery [2].

Even with the most sophisticated and careful methods, ID cannot always be avoided. If encountered and recognized during the procedure, it is broadly accepted that the lesion should be repaired [13]. Besides the gold standard for the management being a primary suture repair $[14,17]$, other techniques have been introduced and are used frequently, including the application of a fat or muscle graft, fascia graft, postoperative blood patch, fibrin glue, or other closure adjuncts such as Gelfoam (Baxter Healthcare Corporation, Hayward, CA, USA), Spongostan (Ethicon Inc., Somerville, NJ, USA), or TachoSil (Takeda Austria GmbH, Linz, Austria) $[18,19]$. Still, the treatment of ID likewise depends on the extent of the durotomy, whether the arachnoid membrane has been opened or remained closed without CSF leakage, and on its location. Especially IDs with deep location in the axilla of the nerve root, or at the anterior dura, may be difficult or impossible to suture. Equally, the treatment of ID differs between classic open and minimally invasive approaches. Concerning the postoperative course, there are, however, no evidence-based or standardized well-established postoperative treatment recommendations.

Moreover, the common complication ID is handled differently among specialties involved in spinal surgery, clinics, and even among individual surgeons at the same institution or department. Traditional management always included flat bed rest for up to many days in order to reduce hydrostatic pressure during the healing process in combination with caffeine and fluid intake to reduce postural headache [20]. The benefit of prolonged postoperative bed rest, however, has been challenged by two recent studies demonstrating no additional benefit and even pointing out a higher complication rate associated with immobilization [20-22].

As considerable controversy exists regarding the management of ID with many different individualized treatment options, the establishment of clear guidelines and recommendations is rather challenging. We were therefore interested in the current management of ID in three different German-speaking countries.

\section{Materials and methods}

In March 2014, members of the Swiss, German, and Austrian neurosurgical and spine societies were invited by electronic mail to complete a practice-based online questionnaire. The online questionnaire (Table 1) comprised 13 questions in order to gain information about the current management of ID of spine surgeons in Switzerland, Germany, and Austria. Questions $1-7$ and 13 were questions with a single response, and questions $8-12$ were questions with multiple responses. Before submission of the questionnaire, a trial survey was tested for usability on a small group of 10 local spine surgeons (data not shown). Two weeks after the first invitation, reminder requests were sent to all invitees, who had not already responded at that time. This was repeated again 4 weeks after the first invitation. Those invitees who did not answer on one of the three invitations were excluded from the final analysis. All responders were assured of confidentiality of the data. IRB approval was not required for this physician survey.

All data were collected in an online database and subsequently exported into SPSS. Frequency distributions and summary statistics were calculated for all questions with categorical answers. Statistical analysis was performed using SPSS (Version 21.0.0.0, SPSS Inc, Chicago, IL, USA) for Windows.

\section{Results}

Responses of all 175/397 participants (overall response rate $44.1 \%$ ) who completed the questionnaire were included into the final data analysis (Table 1).

\section{Demographic characteristics of the participants}

Fifty-nine $(33.7 \%)$ of the respondents were head of departments, 17 (9.7\%) senior consultants, 69 (39.4\%) consultants, and $30(17.1 \%)$ interns/registrars. The majority of the responders were neurosurgeons $(n=157,89.7 \%)$; the others $(n=18,10.3 \%)$ were orthopedic surgeons. Surgeons from Switzerland $(n=108)$ comprised $61.7 \%$ of the included sample, the remainder being from Germany $(n=50,28.6 \%)$ and Austria $(n=17,9.7 \%)$. Seventy $(40.0 \%)$ responders work in a university hospital, while $80(45.7 \%)$ and $31(17.7 \%)$ work in a non-university hospital and private clinic, respectively. Each three orthopedic surgeons and neurosurgeons were working at the same time in a non-university hospital and in a private clinic. Most of the responders $(n=74,42.3 \%)$ perform less than 100 surgical procedures for lumbar disc herniations and/ 
Table 1 Survey questions and responses to evaluate treatment of incidental durotomy

Question Response $\quad n(\%)$

1. What is your position?

2. What is your specialty?

3. In which hospital/clinic do you work?

4. In which country do you work?

5. How many surgical procedures for lumbar disc herniations and/or spinal canal stenosis do you perform yourself each year?

6. Do you systematically register/analyze incidental durotomies?

7. If yes, what is the percentage of incidental durotomies for these procedures?

Intern/Registrar (Assistenzarzt)
Consultant (Beleg-/Oberarzt)
Senior Consultant (Leitender Arzt)
Head of Department (Chefarzt)
Neurosurgeon
Orthopedic Surgeon
University Hospital
Non-university Hospital
Private Clinic
Switzerland
Germany
Austria
$<100$
$100-200$
$>200$
Yes
No

Not systematically registered/analyzed

$0 \%$

$<1 \%$

$1-3 \%$

$3-6 \%$

$6-9 \%$

$>9 \%$

8. What are you doing perioperatively in case of an incidental durotomy?

9. If you are doing something in case of an incidental durotomy, what is it?

10. At the end of the procedure, do you put in an epidural wound drainage (Redon) in case of an incidental durotomy?

Nothing

Only bed rest

Additional action (next question)

Single suture

Running suture

Fibrin glue

Always suture and fibrin glue

Fat tissue patch

Muscle tissue patch

TachoSil/Spongostan with fibrin glue or similar product

Tight closure (layer by layer)

Lumbar drainage (always)

Lumbar drainage (sometimes)

Never

Sometimes

Yes, but only without aspiration

Yes, with aspiration

Never

11. At the end of the procedure, do you prescribe bedrest for your patient in case of an incidental durotomy?
Always bed rest for $24 \mathrm{~h}$

Always bed rest for $48 \mathrm{~h}$

Always bed rest for $72 \mathrm{~h}$

Always bed rest for $>72 \mathrm{~h}$

No bed rest if the incidental durotomy has been closed completely/sufficiently (positive Valsalva)
$30(17.1)$

$69(39.4)$

$17(9.7)$

$59(33.7)$

157 (89.7)

$18(10.3)$

$70(40.0)$

80 (45.7)

$31(17.7)$

108 (61.7)

50 (28.6)

17 (9.7)

74 (42.3)

$60(34.3)$

$41(23.4)$

$74(42.3)$

101 (57.7)

$100(56.8)$

8 (4.5)

35 (19.9)

$22(12.5)$

5 (2.8)

$3(1.7)$

17 (9.7)

34 (19.4)

147 (84.0)

122 (69.7)

47 (26.9)

54 (30.9)

$35(20.0)$

23 (13.1)

36 (20.6)

139 (79.4)

64 (36.6)

$6(3.4)$

$66(37.7)$

65 (37.1)

54 (30.9)

59 (33.7)

18 (10.3)

$26(14.9)$

$61(34.9)$

$49(28.0)$

$11(6.3)$

0 (0)

27 (15.4) 
Table 1 (continued)

\begin{tabular}{|c|c|c|}
\hline Question & Response & $n(\%)$ \\
\hline \multirow{7}{*}{$\begin{array}{l}\text { 12. What are you doing if your patient (with an incidental durotomy) has } \\
\text { persistent signs of a hypoliquorrhea-syndrome (Liquorverlustsyndrom)? }\end{array}$} & $\begin{array}{l}\text { Bed rest if the incidental durotomy could not have been } \\
\text { closed completely/sufficiently (negative Valsalva) }\end{array}$ & $22(12.6)$ \\
\hline & Wait and see & $49(28.0)$ \\
\hline & Lumbar drainage & $35(20.0)$ \\
\hline & Increased per oral fluid intake & $39(22.3)$ \\
\hline & Relative bed rest & $41(23.4)$ \\
\hline & Strict bed rest & $34(19.4)$ \\
\hline & Lumbar MRI to identify source (e.g. CSF fistula) & $96(54.9)$ \\
\hline \multirow{5}{*}{$\begin{array}{l}\text { 13. Do you inform the patient after the operation in case of an incidental } \\
\text { durotomy? }\end{array}$} & Return to the OR for a revision surgery & $80(45.7)$ \\
\hline & Other & $7(4.0)$ \\
\hline & Always & $145(82.9)$ \\
\hline & Sometimes & $28(16.0)$ \\
\hline & Never & $2(1.1)$ \\
\hline
\end{tabular}

or spinal canal stenosis each year, compared to $n=60$ (34.3\%), who perform 100 to 200 procedures, and $n=41$ (23.4\%), who perform more than 200 procedures each year.

\section{Rate and registration of ID}

Most of the responders reported a general percentage of ID of $1-3 \%(n=35,19.9 \%)$ and $3-6 \%(n=22,12.5 \%)$. No significant difference was evident comparing the percentage of ID with specialty $(p=0.097)$, hospital $(p=0.131)$, position $(p=0.064)$, or country $(p=0.524)$. Seventy-four $(42.3 \%)$ responders do systematically register/analyze ID, while there appears to be no differences regarding the country $(p=0.106)$, specialty $(p=0.088)$, or position $(p=0.119)$. However, significantly more responders from non-university hospitals or private clinics $(n=51)$ systematically register/analyze ID compared to 23 responders who work in a university hospital $(p<0.002)$.

\section{General management of ID}

Thirty-four responders (19.4\%) prescribe bed rest only in the case of ID as compared to $147(84.0 \%)$ performing an additional action as described below. Head of departments and senior consultants less frequently prescribe bed rest only in case of ID as compared to interns/registrars and consultants $(p<0.007)$. Less responders from university hospitals or private clinics prescribe bed rest only as compared to responders from non-university hospitals $(p=0.006)$. Significantly fewer responders from Germany $(n=3)$ prescribe bed rest only as compared to responders from Switzerland $(n=19)$ and Austria $(n=12)(p<0.001)$.

\section{Intraoperative management of ID}

Most participants do not rely on bed rest as the only treatment applied, and thus additional actions were declared by 147 (84\%) of the responders. TachoSil/Spongostan with fibrin glue or a similar product $(n=139,79.4 \%)$ and single suture repair $(n=122,69.7 \%)$ are the actions preferred by most surgeons. Here, the application of a single suture does not depend on the position $(p=0.215)$, hospital $(p=0.095)$, or specialty $(p=0.766)$. However, responders from Austria perform a single suture more frequently $(94.1 \%)$ compared to responders from Switzerland $(58.3 \%, p=0.001)$.

Concerning the application of TachoSil/Spongostan with fibrin glue or a similar product, more responders from nonuniversity hospitals $(89.3 \%)$ than from university clinics $(70.0 \%)$ declare its frequent use $(p=0.015)$. Likewise, responders from Germany and Austria use TachoSil/ Spongostan with fibrin glue more frequently than responders from Switzerland $(p<0.011)$, whereas position $(p=0.072)$ and specialty $(p=0.855)$ have no influence on its use. The use of fibrin glue only was not different among the position ( $p=$ $0.079)$, hospital $(p=0.894)$, and specialty $(p=0.436)$, while responders from Germany and Austria used fibrin glue less frequently than responders from Switzerland $(p<0.004)$. Sixty-four (36.6\%) of the responders perform a tight subfascial, fascial, and subcutaneous closure (layer by layer) in case of ID. Here, interns/registrars reported to perform tight closure less frequently than senior consults (20.0 vs. $58.8 \%$, $p=0.026$ ). Additionally, responders from university hospitals more frequently perform a tight closure compared to responders from non-university hospitals $(p=0.022)$.

Concerning epidural wound drainages in case of ID, there is equipoise in the approaches as $37.1 \%$ of responders never place an epidural drainage, whereas $30.9 \%$ sometimes, and $33.7 \%$ regularly place an epidural drainage (without 
aspiration only). No differences were found for responders who never use an epidural drainage and who use an epidural drainage (without aspiration only) comparing position, hospital, specialty, and country $(p>0.102)$. However, responders from non-university hospitals used an epidural drainage with aspiration more frequently compared to responders from university hospitals and private clinics $(p<0.006)$. Additionally, responders from Austria used an epidural drainage with drainage more frequently compared to responders from Switzerland and Germany $(p<0.001)$.

Regarding the occasional application of a lumbar drainage, there were no differences in its use between the position ( $p=$ $0.372)$, hospital $(p=0.053)$, and specialty $(p=0.152)$. However, responders from Austria reported a more frequent occasional application of a lumbar drainage than responders from Switzerland ( 88.2 vs. $26.9 \% p=0.001)$.

\section{Bed rest after ID}

Concerning bed rest, $14.9 \%$ of participants never prescribe bed rest, while 34.9 and $28 \%$ prescribe bed rest always for 24 , respectively always for $48 \mathrm{~h}$. Longer bed rest, e.g. for $72 \mathrm{~h}$ is prescribed less often $(n=11,6.3 \%)$ and no participant responded to recommending more than $72 \mathrm{~h}$ bed rest. There were no differences analyzing the responses that never prescribe bed rest in terms of position $(p=0.068)$, hospital ( $p=$ $0.134)$, specialty $(p=0.241)$, and country $(p=0.332)$. Senior consultants and head of departments prescribed bed rest for $24 \mathrm{~h}$ more frequently compared to interns/registrars and consultants $(p<0.020)$. Also, more responders from Austria prescribed bed rest for $24 \mathrm{~h}$ compared to responders from Germany $(p=0.001)$. However, interns/registrars more frequently prescribed bed rest for $48 \mathrm{~h}$ as compared to consultants (50.0 vs. $23.2 \%, p=0.034$ ) and responders from Switzerland less frequently prescribed bed rest for $72 \mathrm{~h}$ than responders from Germany ( 0.9 vs. $16.0 \%, p=0.001)$. An equal number of participants prescribed no bed rest if the ID has been closed completely/sufficiently (positive Valsalva) $(n=27,15.4 \%)$ or if the ID could not have been closed completely/sufficiently (negative Valsalva) $(n=22,12.6 \%)$.

\section{Management of hypoliquorrhea syndrome}

In case of persistent signs of a hypoliquorrhea syndrome in a patient after ID, most participants of the survey perform a lumbar MRI to identify the source (e.g., CSF fistula) $(n=96$, $54.9 \%)$ or return to the OR for a revision surgery $(n=80$, $45.7 \%$ ). Here, especially interns/registrars as compared to more senior consultants $(p=0.001)$ and responders from Austria as compared to responders from Switzerland and Germany $(p<0.014)$ prefer to perform a lumbar MRI to identify the source, while responders from private clinics do so less frequently when compared to responders from university or non- university hospitals $(p<0.001)$. No differences were found for the response "Return to the OR for a revision surgery" comparing the position, type of hospital, specialty, and country $(p>0.089)$. Equally, there were no differences for the responses "Wait and see", "Lumbar drainage", and "Increased per oral fluid intake" comparing the position, type of hospital, specialty, and country $(p=0.074)$. However, in case of a postoperative hypoliquorrhoe syndrome, interns/registrars prescribed strict bed rest more frequently $(p=0.004)$ and relative bed rest less frequently as compared to senior consultants $(p=0.008)$. Responders from Austria likewise prescribed relative bed rest less frequently as compared to responders from Germany $(p=0.019)$.

\section{Communication of ID}

The vast majority of the responders ( $n=145,82.9 \%$ ) always inform their patients in case of ID after the operation, whereas 28 responders $(16.0 \%)$ inform their patients sometimes, and two responders $(1.1 \%)$ never inform their patients. There were no differences for the response "Always" comparing positions, hospitals, and countries $(p=0.573)$. Of note, $100 \%$ of the orthopedic surgeons $(n=18)$ reported to always inform their patients in case of ID as compared to $80.9 \%(127 /$ 157 ) of neurosurgeons $(p=0.042)$. Lastly, there were no differences for the response "Sometimes" comparing position, hospital, specialty, and country $(p=0.051)$.

\section{Discussion}

The current survey shows that there is substantial heterogeneity in the management of IDs throughout the Germanspeaking European countries. This reflects the fact that there are, so far, no standardized and well-established intra- and postoperative treatment recommendations. Nonetheless, it is generally accepted that all IDs should be primarily repaired [13], which is also depicted by the results of our survey with the majority of the survey responders using single sutures and TachoSil/Spongostan with fibrin glue or a similar product in order to achieve a primary repair. Other options, like bed rest, fibrin glue, fat or muscle tissue patch, tight closure, and/or a lumbar drainage, were used in variable fashion in order to achieve and maintain permanent closure of the ID. Only $9.7 \%$ of the survey responders responded that they would not undertake anything particular perioperatively in case of ID.

The fact that significantly fewer responders from Germany as compared to their Swiss colleagues prescribed bed rest only in case of ID is most likely an effect of the respective surgical training program and the individual school of the predominant personal tutor. Although traditionally every country had its own individual training program (with a certain exchange), 
there are on-going efforts to establish European training standards [23-25]. Besides hospital's policy or surgeon's preference, the financial aspect and the reimbursement policy play a role which and how many so-called closure adjuncts might be used. The current survey revealed that $14.9 \%(n=26)$ of the responders never prescribe bed rest in case of ID, whereas the majority, namely $34.9 \%(n=61)$ and $28 \%(n=49)$ prescribe bed rest always for 24 and $48 \mathrm{~h}$, respectively. The traditional recommendation of $72 \mathrm{~h}$ of flat bed rest in case of ID was only reported by $6.3 \%(n=11)$ of the responders, and bed rest for more than $72 \mathrm{~h}$ seems to be no option anymore today. Interestingly, more senior consultants and head of departments favored bed rest for $24 \mathrm{~h}$ as compared to interns/registrars and consultants. On the other hand, bedrest for $48 \mathrm{~h}$ was more frequently prescribed by interns/registrars as compared to all other positions. We interpret this finding with a higher confidence in senior surgeons (due to their longer experience) that $24 \mathrm{~h}$ of bed rest is sufficient to avoid further complications.

Also internationally accepted, bed rest after ID remains a highly debated issue. Radcliff et al. were the first to report on complications of flat bed rest in specific association with ID [20]. In a retrospective case series of consecutive patients with ID following lumbar laminectomy between 2005 and 2009, the authors compared $n=24$ patients with prolonged bed rest $>24 \mathrm{~h}$ and $n=18$ patients with a duration of bed rest $\leq 24 \mathrm{~h}$. While the rates of postdurotomy-related neurological complications, wound complications, and need for revision surgery were similar, the authors found a significantly increased rate of total medical complications (pneumonia, deep vein thrombosis) in the group with prolonged bed rest ( 0 vs. $50 \%, p=0.0003)$. The authors concluded that flat bed rest after sufficient dural repair should be avoided, as it may lead to a higher incidence of medical complications. These findings correspond with complications of prolonged bed rest reported in the medical literature including thromboembolic disease, wound infection, and pulmonary and gastrointestinal complications $[20,26]$. Low et al. likewise reported on the incidence of complications in patients with ID after lumbar spine surgery [21]. Twenty-six patients were mobilized on the first postoperative day, nine patients on the second, and the remaining 26 patients were mobilized on the third postoperative day or later. The overall incidence of durotomy-related complications was $18 \%$. The authors found no association between the day of mobilization and the rate of complications ( $p=0.433)$ and therefore advocate allowing postoperative mobilization as soon as possible. There are several studies about the management of ID without mandatory bed rest. In their retrospective review of 20 patients with ID treated without mandatory bed rest, Hodges et al. found that, after primary repair with dural stitches and fibrin glue, $75 \%$ of patients had no symptoms [27]. It should be pointed out, however, that the dural tears in their study were relatively small, with a length of $1-3 \mathrm{~mm}$. Than et al. reported on a limited series of five patients who underwent minimally invasive lumbar spine surgery complicated by ID [28]. Following primary repair including the application of a closure adjunct, all patients were mobilized in less than $48 \mathrm{~h}$ after surgery. Two patients were mobilized after recovery from anesthesia, two patients were mobilized the morning after surgery, and one patient was mobilized early on the second postoperative day. None of the five patients developed durotomy-related symptoms. Ohana and Kleir briefly presented their experience with immediate mobilization following incidental durotomy after spine surgery [22]. A complete closure of the durotomy was possible in ten out of 13 patients while in the remaining cases a fibrin glue sealing procedure was performed. All except for one patient had a regular immediate postoperative mobilization without any late CSF leakage or other related complications.

In our opinion, the question if the treating surgeon does systematically register/analyze IDs deserves specific attention. Interestingly, we found significant differences with $32.9 \%(n=$ 23) of responders who are working in a university hospital, up to $40 \%(n=30)$ and $70.0 \%(n=21)$ of responders who are working in a non-university hospital and in a private clinic, respectively. As most spine registries have been developed and launched in university hospitals and quality control is gaining increasing importance, this finding is somehow controversial [29-31]. However, a possible explanation for this finding is that the documentation and outcome assessment might be even more meticulous in non-university hospitals and private clinics today in order to accurately document the surgical results and outcomes as a quality control. As quality control is of paramount importance for our health care systems, if not already established, we recommend the implementation of systematic registration systems for all hospitals performing spine surgery.

\section{Strengths and limitations}

For the analysis of certain subgroups, especially the comparisons of the different countries and the surgical specialties have to be interpreted with caution, as there are low absolute numbers in the group of orthopedic surgeons $(n=18,10.3 \%)$ and physicians from Austria $(n=17,9.7 \%)$. On the other hand, a similar amount of responders to our survey reported to perform less than $100(42.3 \%), 100-200(34.3 \%)$, or less than 200 procedures per year $(23.4 \%)$, indicating that our results represent the current management performed by a representative sample of spine surgeons with all levels of expertise. Another limitation is the relatively low response rate of $44.1 \%$ and the subgroup of interns/registrars (accounting for $17.1 \%$ of the participants) with correspondent less surgical experience. Additionally, we did not differentiate in the online survey if the ID is with or without CSF flow, as there is a subgroup of IDs with a remaining arachnoid layer omitting liquor flow. Therefore, we asked a subgroup of participants, all of them confirming that they understood and answered the questions, as the ID would come along with liquor flow. These observations are altogether 
very reasonable, as the surgical activity mainly depends on the respective surgeon's position and experience. Moreover, it depends on the hospital's caseload, the specific subspecialty, and diversity of the corresponding surgeon. The plausibility of these observations demonstrates that the responses received in this survey are stable, represent the real clinical setting in Germanspeaking countries today, and are not biased by certain overrepresented subgroups in our survey.

Implications on the management of ID

In the absence of evidence from randomized controlled trials and consensus statement from appropriate authorities we recommend, in accordance with the above data, the direct intraoperative repair of the dural defect. The repair should be either with a single suture if the defect is small, or with a continuous suture if the defect is larger. Additionally, if possible from an anesthesiological point of view, we try to put the patient in a Trendelenburg position in order to avoid further CSF leakage. If a subsequent primary closure is not possible, consider a local autograft with a paraspinal muscle patch with or without additional closure aids, according to the individual local situation (e.g., TachoSil, Gelfoam/Spongostan, or fibrin glue). Before wound closure, a Valsalva maneuver could be used to test the integrity of the durotomy repair. Furthermore, the dura should re-inflate in a pulsatile fashion, confirming that a watertight closure has been achieved. If no more CSF leak is evident, the wound is to be tightly closed layer by layer. We recommend against the use of regular postoperative epidural wound drains in case of ID (surgeon judgment is advocated in this situation) on the basis of lack of evidence of benefit, and known risks (e.g., infections, subdural hematomas, CSFfistulas) which is deemed appropriate given the findings of this study, where only six (3.4\%) surgeons surveyed described the regular use of a post-operative drainage. However, if hemostasis is not completely possible, and the risk (as assessed by the treating surgeon) of postoperative epidural hemorrhage is expected to be higher than the risk of persistent CSF fistula, a wound drain without suction should be used. The role of postoperative bed rest has been challenged considering the latest literature reports (see above).

\section{Conclusions}

There is substantial heterogeneity in the management of incidental durotomies in European countries today. Most of the participants perform primary closure with or without the application of fibrin glue or other closure adjuncts. The recommendation of bed rest varies largely between no bed rest, short- to mid-term bed rest over 24 to $48 \mathrm{~h}$, whereas a trend towards no bed rest and early mobilization is observed if the
ID has been closed completely. As ID represents one of the most common complications in lumbar spine surgery with possible negative influence on the mid- and long-term patient outcome, further research is needed to establish evidencebased recommendations, and also to finally define the role of (prolonged) bed rest in case of ID.

Acknowledgments The authors of this work thank all participants for having answered this survey.

Conflict of interest None.

\section{References}

1. Deyo RA, Cherkin DC, Loeser JD, Bigos SJ, Ciol MA (1992) Morbidity and mortality in association with operations on the lumbar spine. The influence of age, diagnosis, and procedure. J Bone Joint Surg Am 74:536-543

2. Kalevski SK, Peev NA, Haritonov DG (2010) Incidental dural tears in lumbar decompressive surgery: incidence, causes, treatment, results. Asian J Neurosurg 5:54-59

3. Tafazal SI, Sell PJ (2005) Incidental durotomy in lumbar spine surgery: incidence and management. Eur Spine J 14:287-290

4. Baker GA, Cizik AM, Bransford RJ, Bellabarba C, Konodi MA, Chapman JR, Lee MJ (2012) Risk factors for unintended durotomy during spine surgery: a multivariate analysis. Spine J 12:121-126

5. Jankowitz BT, Atteberry DS, Gerszten PC, Karausky P, Cheng BC, Faught R, Welch WC (2009) Effect of fibrin glue on the prevention of persistent cerebral spinal fluid leakage after incidental durotomy during lumbar spinal surgery. Eur Spine J 18:1169-1174

6. Khan MH, Rihn J, Steele G, Davis R, Donaldson WF 3rd, Kang JD, Lee JY (2006) Postoperative management protocol for incidental dural tears during degenerative lumbar spine surgery: a review of 3 , 183 consecutive degenerative lumbar cases. Spine 31:2609-2613

7. McMahon P, Dididze M, Levi AD (2012) Incidental durotomy after spinal surgery: a prospective study in an academic institution. J Neurosurg Spine 17:30-36

8. Stromqvist F, Jonsson B, Stromqvist B, Swedish Society of Spinal S (2010) Dural lesions in lumbar disc herniation surgery: incidence, risk factors, and outcome. Eur Spine J 19:439-442

9. Williams BJ, Sansur CA, Smith JS, Berven SH, Broadstone PA, Choma TJ, Goytan MJ, Noordeen HH, Knapp DR, Hart RA, Zeller RD, Donaldson WF, Polly DW, Perra JH, Boachie-Adjei O, Shaffrey CI (2011) Incidence of unintended durotomy in spine surgery based on 108,478 cases. Neurosurgery $68: 117-123$

10. Desai A, Ball PA, Bekelis K, Lurie J, Mirza SK, Tosteson TD, Weinstein JN (2011) SPORT: does incidental durotomy affect longterm outcomes in cases of spinal stenosis? Neurosurgery 69:38-44

11. Sin AH, Caldito G, Smith D, Rashidi M, Willis B, Nanda A (2006) Predictive factors for dural tear and cerebrospinal fluid leakage in patients undergoing lumbar surgery. J Neurosurg Spine 5:224-227

12. Takahashi Y, Sato T, Hyodo H, Kawamata T, Takahashi E, Miyatake N, Tokunaga M (2013) Incidental durotomy during lumbar spine surgery: risk factors and anatomic locations: clinical article. J Neurosurg Spine 18:165-169

13. Guerin P, El Fegoun AB, Obeid I, Gille O, Lelong L, Luc S, Bourghli A, Cursolle JC, Pointillart V, Vital JM (2012) Incidental durotomy during spine surgery: incidence, management and complications. A retrospective review. Injury 43:397-401 
14. Cammisa FP, Girardi FP, Sangani PK, Parvataneni HK, Cadag S, Sandhu HS (2000) Incidental durotomy in spine surgery. Spine 25: 2663-2667

15. Desai A, Ball PA, Bekelis K, Lurie J, Mirza SK, Tosteson TD, Zhao W, Weinstein JN (2012) Surgery for lumbar degenerative spondylolisthesis in spine patient outcomes research trial: does incidental durotomy affect outcome? Spine 37:406-413

16. Desai A, Ball PA, Bekelis K, Lurie JD, Mirza SK, Tosteson TD, Weinstein JN (2011) Outcomes after incidental durotomy during first-time lumbar discectomy. J Neurosurg Spine 14:647-653

17. Wang JC, Bohlman HH, Riew KD (1998) Dural tears secondary to operations on the lumbar spine. Management and results after a twoyear-minimum follow-up of eighty-eight patients. J Bone Joint Surg Am 80:1728-1732

18. Espiritu MT, Rhyne A, Darden BV (2010) Dural tears in spine surgery. J Am Acad Orthop Surg 18:537-545

19. Miscusi M, Polli FM, Forcato S, Coman MA, Ricciardi L, Ramieri A, Raco A (2014) The use of surgical sealants in the repair of dural tears during non-instrumented spinal surgery. Eur Spine J. 2014. PMID 24384831

20. Radcliff KE, Sidhu GD, Kepler CK, Gruskay J, Anderson DG, Hilibrand A, Albert TJ, Vaccaro AR (2013) Complications of flat bedrest following incidental dural repair. J Spinal Disord Tech. PMID 23197257

21. Low JC, von Niederhausern B, Rutherford SA, King AT (2013) Pilot study of perioperative accidental durotomy: does the period of postoperative bed rest reduce the incidence of complication? Br J Neurosurg 27:800-802

22. Ohana N, Kleir I (2005) Immediate mobilization following incidental durotomy in spine surgery. J Bone Joint Surg (Br) 87-B:379
23. Eroes CA, Barth C, Tonn JC, Reulen HJ (2008) The revised European neurosurgical electronic logbook of operations. Acta Neurochir (Wien) 150:195-198

24. Lindsay K (2012) Accreditation of neurosurgical training programmes in Europe: report of JRAAC. Acta Neurochir (Wien) 154:947-949

25. Omerhodzic I, Tonge M, Matos B, Musabeliu E, Raspanti C, Ferdinandov D, Galimova R, Muroi C, Balik V, Kursumovic A (2012) Neurosurgical training programme in selected European countries: from the young neurosurgeons' point of view. Turk Neurosurg 22:286-293

26. Allen C, Glasziou P, Del Mar C (1999) Bed rest: a potentially harmful treatment needing more careful evaluation. Lancet 354:1229-1233

27. Hodges SD, Humphreys SC, Eck JC, Covington LA (1999) Management of incidental durotomy without mandatory bed rest. A retrospective review of 20 cases. Spine 24:2062-2064

28. Than KD, Wang AC, Etame AB, La Marca F, Park P (2008) Postoperative management of incidental durotomy in minimally invasive lumbar spinal surgery. Minim Invasive Neurosurg MIN 51:263-266

29. Aebi M, Grob D (2004) SSE spine tango: a European spine registry promoted by the Spine Society of Europe (SSE). Eur Spine J 13:661662

30. Porchet F, Bartanusz V, Kleinstueck FS, Lattig F, Jeszenszky D, Grob D, Mannion AF (2009) Microdiscectomy compared with standard discectomy: an old problem revisited with new outcome measures within the framework of a spine surgical registry. Eur Spine J 18(Suppl 3):360-366

31. Schluessmann E, Diel P, Aghayev E, Zweig T, Moulin P, Roder C, Group SWR (2009) SWISSspine: a nationwide registry for health technology assessment of lumbar disc prostheses. Eur Spine J 18:851-861 